ARTICLE

\title{
A highly active and stable hydrogen evolution catalyst based on pyrite-structured cobalt phosphosulfide
}

Wen Liu', Enyuan $\mathrm{Hu}^{2}$, Hong Jiang ${ }^{3}$, Yingjie Xiang ${ }^{4}$, Zhe Weng ${ }^{1}$, Min Li ${ }^{5}$, Qi Fan ${ }^{1}$, Xiqian Yu ${ }^{2}$ Eric I. Altman ${ }^{5}$ \& Hailiang Wang ${ }^{1}$

Rational design and controlled synthesis of hybrid structures comprising multiple components with distinctive functionalities are an intriguing and challenging approach to materials development for important energy applications like electrocatalytic hydrogen production, where there is a great need for cost effective, active and durable catalyst materials to replace the precious platinum. Here we report a structure design and sequential synthesis of a highly active and stable hydrogen evolution electrocatalyst material based on pyrite-structured cobalt phosphosulfide nanoparticles grown on carbon nanotubes. The three synthetic steps in turn render electrical conductivity, catalytic activity and stability to the material. The hybrid material exhibits superior activity for hydrogen evolution, achieving current densities of $10 \mathrm{~mA} \mathrm{~cm}^{-2}$ and $100 \mathrm{~mA} \mathrm{~cm}^{-2}$ at overpotentials of $48 \mathrm{mV}$ and $109 \mathrm{mV}$, respectively. Phosphorus substitution is crucial for the chemical stability and catalytic durability of the material, the molecular origins of which are uncovered by X-ray absorption spectroscopy and computational simulation.

\footnotetext{
${ }^{1}$ Department of Chemistry and Energy Sciences Institute, Yale University, 520 West Campus Drive, West Haven, Connecticut 06511, USA. ${ }^{2}$ Chemistry Department, Brookhaven National Laboratory, Upton, New York 11973, USA. ${ }^{3}$ Beijing National Laboratory for Molecular Sciences, College of Chemistry and Molecular Engineering, Peking University, Beijing 100871, China. ${ }^{4}$ Department of Mechanical Engineering and Materials Science, Yale University, 520 West Campus Drive, West Haven, Connecticut 06511, USA. ${ }^{5}$ Department of Chemical and Environmental Engineering, Yale University, 520 West Campus Drive, West Haven, Connecticut 06511, USA. Correspondence and requests for materials should be addressed to H.W. (email: hailiang.wang@yale.edu).
} 
W ith the rising concern over energy crisis and environmental pollution, there has been a growing need to replace fossil fuels with clean and sustainable energy carriers. Molecular hydrogen, with its high energy density and non-polluting characteristics, has been regarded as one of the most promising new fuels ${ }^{1,2}$. There are various methods to produce hydrogen, among which water splitting driven by electricity generated from renewable energy sources is an attractive way to support the future hydrogen economy. Efficient electrolytic hydrogen generation relies heavily on active, durable and affordable catalysts to accelerate the kinetics ${ }^{3-5}$. Although platinum (Pt) has been widely acknowledged as the most active catalyst for the hydrogen evolution reaction (HER), this precious metal is scarce in the earth's crust and expensive for large scale applications.

In pursuit of inexpensive replacements for $\mathrm{Pt}$ as the HER electrocatalyst in acidic solutions, numerous inorganic materials based on non-precious transition metals, including sulfides ${ }^{6-8}$, selenides $^{9,10}$, phosphides ${ }^{11-13}$ and others ${ }^{14,15}$ have been explored. Extensive effort has been devoted to improving the HER catalytic activity of molybdenum disulfide $\left(\mathrm{MoS}_{2}\right)$ by identifying and exposing active sites ${ }^{6,16}$, as well as enhancing electron conduction through nanostructuring $^{17}$, shape control ${ }^{6,18}$, phase engineering ${ }^{19,20}$, doping $^{21,22}$, intercalation ${ }^{23}$ and hybridization ${ }^{17,24,25}$. Recently a ternary cobalt phosphosulfide pyrite-type structure (CoPS) has been reported with superior catalytic activity for $\mathrm{HER}^{26}$. Cobalt disulfide $\left(\mathrm{CoS}_{2}\right)$ is another material of interest ${ }^{27,28}$. However, the existing HER catalyst materials based on non-noble metal elements are still less satisfactory in terms of both activity and stability, which calls for further material structure innovation to realize efficient and cost effective HER catalysis.

Here we report a design and synthesis of a highly active and stable HER electrocatalyst material consisting of pyrite-structured cobalt phosphosulfide $(\mathrm{CoS} \mid \mathrm{P})$ nanoparticles anchored on carbon nanotubes (CNTs). The material architecture is built by a three-step chemical synthesis: Strong interactions with CNTs and particle size control are first established by the selective growth of cobalt(II,III) oxide $\left(\mathrm{Co}_{3} \mathrm{O}_{4}\right)$ nanoparticles on CNTs; High catalytic activity for HER is then rendered by conversion of $\mathrm{Co}_{3} \mathrm{O}_{4}$ to $\mathrm{CoS}_{2}$ nanoparticles; Good chemical stability and catalytic durability are lastly obtained from substituting some of the sulfur with phosphorus. The unique material structure directly enables some of the highest HER catalytic performance among all Co-based catalyst materials. In $0.5 \mathrm{M} \mathrm{H}_{2} \mathrm{SO}_{4}$, the $\mathrm{CoS} \mid \mathrm{P} / \mathrm{CNT}$ hybrid exhibits a negligible onset overpotential and a Tafel slop of $55 \mathrm{mV}$ per decade with an exchange current density of $1.14 \mathrm{~mA} \mathrm{~cm}^{-2}$. At a mass loading of $1.6 \mathrm{mg} \mathrm{cm}^{-2}$, the hybrid material requires overpotentials of only $48 \mathrm{mV}$ and $109 \mathrm{mV}$ to reach stable catalytic current densities of $10 \mathrm{~mA} \mathrm{~cm}^{-2}$ and $100 \mathrm{~mA} \mathrm{~cm}^{-2}$ respectively, representing one of the few most active non-Pt catalysts for HER. Phosphorus substitution in the pyrite structure is a critical step that renders chemical stability and catalytic durability to the CoS $\mid \mathrm{P} / \mathrm{CNT}$ hybrid material. Density functional theory (DFT) calculations confirm the structural stability of pyrite $\operatorname{CoS} \mid \mathrm{P}$ and suggest stronger metal-ligand bonding as a contributor to the improved stability, which is supported by X-ray absorption spectroscopy data.

\section{Results}

Sequential synthesis of $\mathrm{CoS} \mid \mathrm{P} / \mathrm{CNT}$. The synthetic strategy for the $\mathrm{CoS} \mid \mathrm{P} / \mathrm{CNT}$ hybrid material structure involves three steps, as illustrated in Fig. 1. In the first step, $\mathrm{Co}_{3} \mathrm{O}_{4}$ nanoparticles were directly and selectively grown onto mildly oxidized multi-wall CNTs (see Supplementary Methods for CNT oxidation) by a hydrolysis reaction of cobalt acetate at $80^{\circ} \mathrm{C}$. An ethanol/water mixed solvent was used to slow down the hydrolysis reaction, facilitate interactions between the $\mathrm{Co}^{2+}$ and the functional groups on the CNT surface, and ensure selective nucleation and growth of $\mathrm{Co}_{3} \mathrm{O}_{4}$ nanoparticles on CNTs. $\mathrm{NH}_{3} \cdot \mathrm{H}_{2} \mathrm{O}$ was added into the reaction system to coordinate the $\mathrm{Co}^{2+}$ and thus further reduce the hydrolysis rate to limit the size of the resulting $\mathrm{Co}_{3} \mathrm{O}_{4}$ nanoparticles and optimize their distribution on the CNTs. Scanning electron microscopy (SEM), transmission electron microscopy (TEM) and X-ray diffraction (XRD) characterizations revealed the product structure as spinel structured $\mathrm{Co}_{3} \mathrm{O}_{4}$ (PDF\#43-1003) nanoparticles with an average size of $5-10 \mathrm{~nm}$ anchored on CNTs (Supplementary Fig. 1).

The second step is a hydrothermal reaction at $200{ }^{\circ} \mathrm{C}$ to convert $\mathrm{Co}_{3} \mathrm{O}_{4}$ into $\mathrm{CoS}_{2}$. Thioacetamide $\left(\mathrm{CH}_{3} \mathrm{CSNH}_{2}\right)$ was used as a slowrelease $\mathrm{S}$ precursor, which reacted with water to gradually generate the $\mathrm{H}_{2} \mathrm{~S}$ reactant and therefore allowed for facile chemical conversion from oxide to sulfide without damaging the material morphology and hybrid structure. SEM and TEM imaging showed nanoparticles with an average size of $10-20 \mathrm{~nm}$ attached to CNTs (Supplementary Fig. 2a,b). The nanoparticles were confirmed to be pyrite-structured $\mathrm{CoS}_{2}$ (PDF\#41-1471) by XRD (Supplementary Fig. 2d). Lattice fringes of the $\mathrm{CoS}_{2}$ nanoparticles on CNTs were recorded with high-resolution TEM (Supplementary Fig. 2c). Energy dispersive spectroscopy (EDS) under scanning TEM (STEM) mode was used to map the distributions of elements of interest in the material structure. It is clear from the result that the
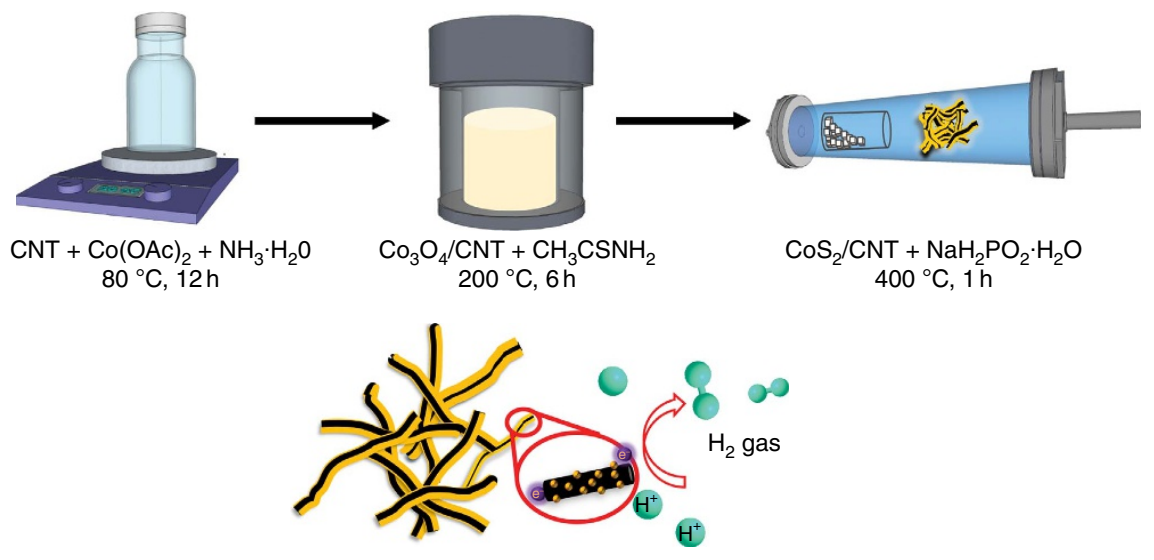

CoS|P/CNT hybrid for HER

Figure 1 | Schematic illustration of the sequential synthesis of the CoS|P/CNT hybrid material for HER catalysis. The CoS|P/CNT is synthesized through three steps including hydrolysis, hydrothermal sulfurization and solid/gas-phase phosphorization. 
nanoparticles consist of Co and S, and they are well-anchored on the CNT surface (Supplementary Fig. 3).

The third step features a solid/gas-phase reaction at $400^{\circ} \mathrm{C}$ to introduce $\mathrm{P}$ into the $\mathrm{CoS}_{2}$ structure. $\mathrm{NaH}_{2} \mathrm{PO}_{2} \cdot \mathrm{H}_{2} \mathrm{O}$ was used as a precursor to generate the $\mathrm{PH}_{3}$ reactant via thermal decomposition. The $\mathrm{PH}_{3}$ then reacted with the $\mathrm{CoS}_{2}$ nanoparticles on CNTs to form the final $\mathrm{CoS} / \mathrm{P} / \mathrm{CNT}$ hybrid material. SEM and TEM characterizations confirmed the microstructure of nanoparticles with an average size of $10-20 \mathrm{~nm}$ on CNTs (Fig. 2a,b), suggesting that the substitution process had negligible influence on the material morphology and nanoparticle size. XRD measurement of the material generated a diffraction pattern characteristic of a pyrite structure with almost identical lattice parameters as $\mathrm{CoS}_{2}$ (Fig. 2c), indicating that the substitution process happened in the form of $\mathrm{P}$ replacing $\mathrm{S}$, which did not alter the crystal structure or lattice parameters due to the very similar atomic sizes of $\mathrm{S}$ and $\mathrm{P}$. The lattice fringes of the $\operatorname{CoS} \mid \mathrm{P}$ nanoparticles were imaged by high-resolution TEM (Fig. 2d). The interplanar spacing of $0.277 \mathrm{~nm}$ corresponding to the (200) crystallographic planes of the pyrite structure is in consistency with the XRD result. The high-resolution TEM images also excluded the existence of core-shell structured nanoparticles.

Structural and chemical analysis. To further understand the structure of the CoS $\mid \mathrm{P} / \mathrm{CNT}$ hybrid material, STEM-EDS characterization was performed to gain elemental composition and distribution information. The EDS maps of Co, S and P overlap quite well (Fig. 3a,b), suggesting $\mathrm{P}$ has been uniformly doped into the crystal structure of nanoparticles. It is also evident from the EDS mapping that the CoS $\mid \mathrm{P}$ nanoparticles are closely anchored onto the CNT surfaces. The average atomic ratio of $\mathrm{P} / \mathrm{S}$ in the nanoparticles was calculated to be $\sim 1.0$ from the EDS spectrum (Fig. $3 \mathrm{c}$ and Supplementary Fig. 4), giving a 50\% substitution of the $S$ sites. Raman spectroscopy was used to probe chemical bonding information in the $\operatorname{CoS} \mid \mathrm{P}$ nanoparticles. For the $\mathrm{CoS}_{2} / \mathrm{CNT}$, two peaks were observed at 284 and $385 \mathrm{~cm}^{-1}$
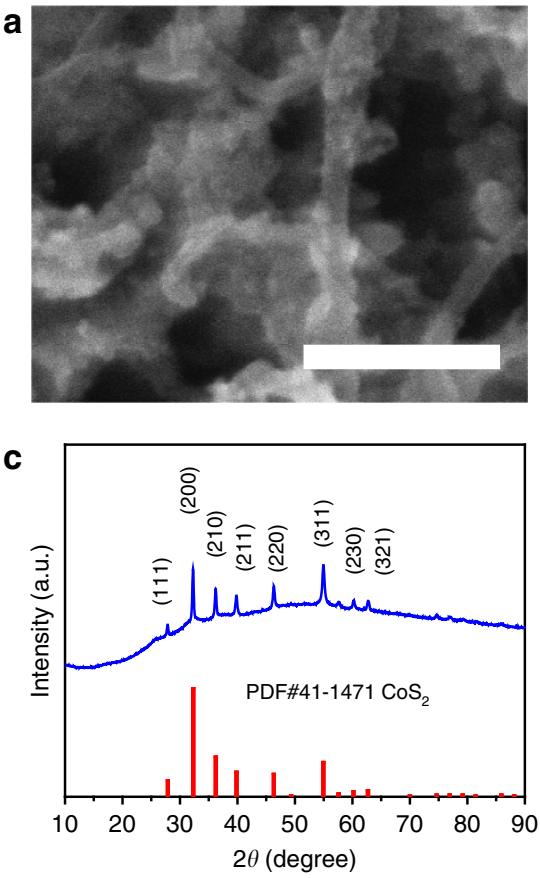

(Fig. 3d, blue trace). These Raman peaks could be attributed to the characteristic $E_{\mathrm{g}}$ and $A_{\mathrm{g}}$ vibrational modes corresponding to the in-phase stretching and pure libration of the S-S dumbbells in the pyrite structure ${ }^{29-31}$. For the $\operatorname{CoS} \mid \mathrm{P} / \mathrm{CNT}$, the peaks slightly shifted to higher wavenumber (Fig. 3d, red trace) as a result of partial phosphorus substitution for sulfur and possible formation of P-S dumbbells. The characteristic Raman pattern also excludes formation of $\mathrm{CoP}^{32}$.

X-ray photoelectron spectroscopy (XPS) was employed to investigate the surface composition and oxidation states of the catalysts. For the $\mathrm{CoS}_{2} / \mathrm{CNT}$, Co $2 p_{3 / 2}$ and $2 p_{1 / 2}$ core level peaks were observed at binding energies of $778.8 \mathrm{eV}$ and $794.0 \mathrm{eV}$, respectively, together with satellite features (Fig. 3e), which match literature results on $\mathrm{CoS}_{2}$ (ref. 28). The Co $2 p$ core level spectrum of the $\mathrm{CoS} \mid \mathrm{P} / \mathrm{CNT}$ hybrid is very similar to that of the $\mathrm{CoS}_{2} / \mathrm{CNT}$, with binding energies of $\mathrm{Co} 2 p_{3 / 2}$ and $2 p_{1 / 2}$ peaks at $779.2 \mathrm{eV}$ and $794.1 \mathrm{eV}$, respectively (Fig. 3e). The negligible change in the Co $2 p$ spectrum verifies that the oxidation state of $\mathrm{Co}$ is not affected by P substitution. The S $2 p$ core level spectrum of the $\mathrm{CoS}_{2} / \mathrm{CNT}$ shows lower binding energy components at $162.6 \mathrm{eV}$ and $163.9 \mathrm{eV}\left(\mathrm{S} 2 p_{3 / 2}\right.$ and $2 p_{1 / 2}$ ) attributed to sulfide species as well as higher binding energy components at $168.5 \mathrm{eV}$ and $169.6 \mathrm{eV}$ ( $2 p_{3 / 2}$ and $2 p_{1 / 2}$ ), respectively, characteristic of sulfate species (Fig. 3f). Existence of the sulfate components suggests that the $\mathrm{CoS}_{2} / \mathrm{CNT}$ catalyst is slightly oxidized on surface. Interestingly, no sulfate features were found in the $\mathrm{S} 2 p$ core level spectrum of the $\mathrm{CoS} / \mathrm{P} / \mathrm{CNT}$ (Fig. 3f), indicating P doping could prevent $\mathrm{CoS}_{2}$ from oxidation under ambient conditions. The $\mathrm{P} 2 p$ core level spectrum of the $\mathrm{CoS} / \mathrm{P} / \mathrm{CNT}$ displays two peak regions (Fig. 3g), with one centred at the binding energy of 129.3 and $130.1 \mathrm{eV}$ ( $\mathrm{P} 2 p_{3 / 2}$ and $2 p_{1 / 2}$ ), which can be assigned to phosphorus anions, and the other at 133.6 and $134.4 \mathrm{eV}$ (unresolved doublet) characteristic of phosphate-like P. The existence of the high oxidation state $\mathrm{P}$ could be ascribed to surface oxidation under ambient conditions as often observed for metal phosphide materials ${ }^{33-35}$. Furthermore, a surface $\mathrm{P} / \mathrm{S}$ ratio of $\sim 1.0$ was derived from the XPS results, close to the bulk $\mathrm{P} / \mathrm{S}$ ratio measured
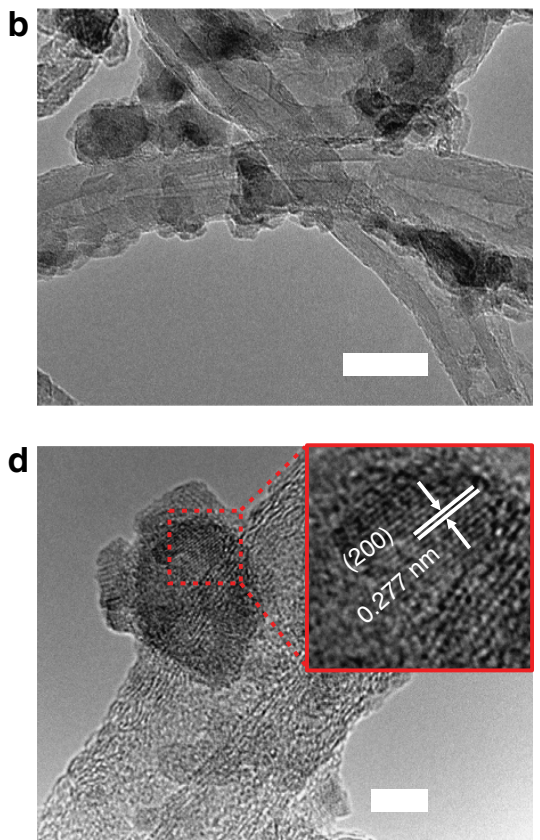

Figure 2 | Structural characterizations of the CoS|P/CNT hybrid material. (a) SEM image of CoS|P/CNT; Scale bar, $200 \mathrm{~nm}$. (b) Low-magnification TEM image of CoS|P/CNT showing nanoparticles attached to CNTs; Scale bar, $20 \mathrm{~nm}$. (c) XRD pattern of CoS|P/CNT as compared with the pyrite-phase CoS 2 standard (PDF\#41-1471). (d) High-resolution TEM image showing the (200) lattice fringes of pyrite-phase CoS|P; Scale bar, $5 \mathrm{~nm}$. 
a

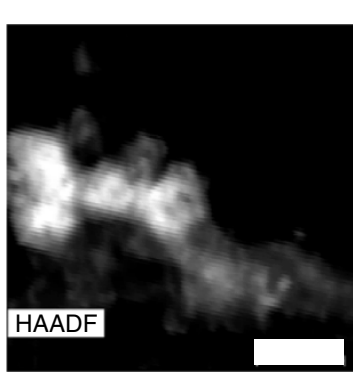

C
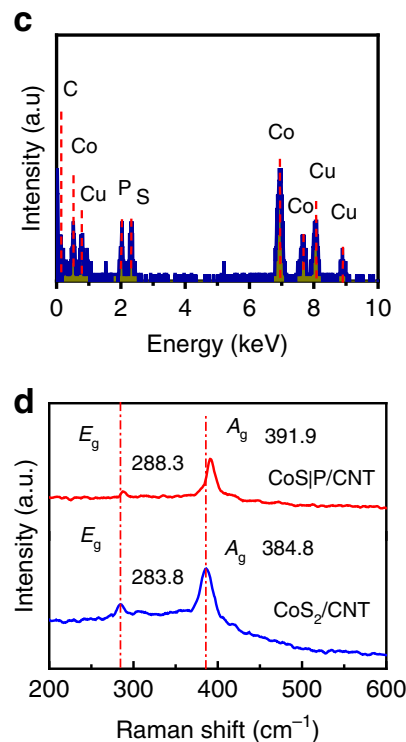

b

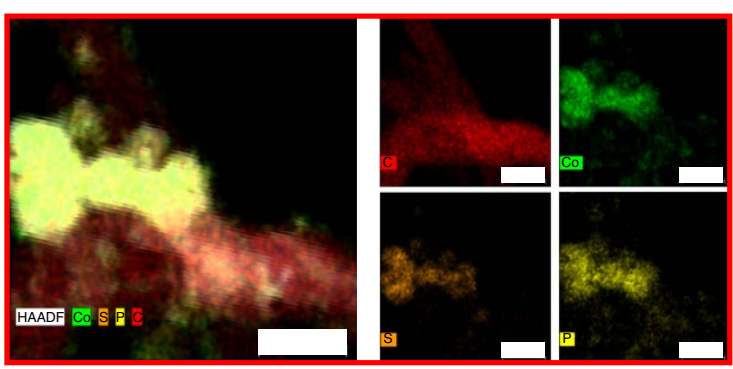

e

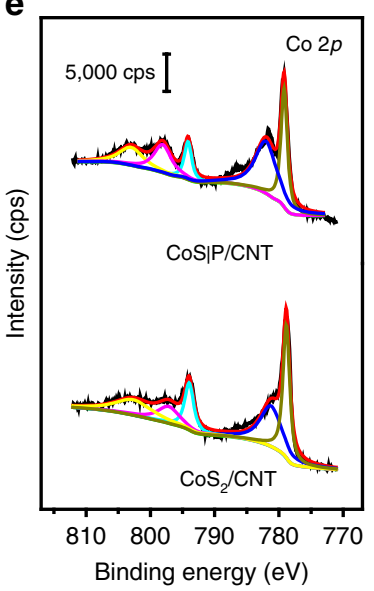

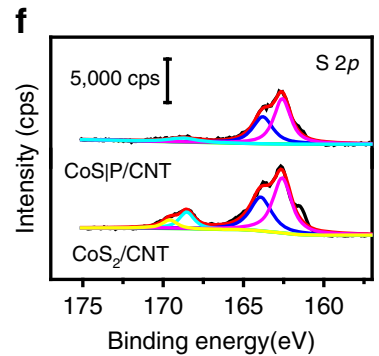

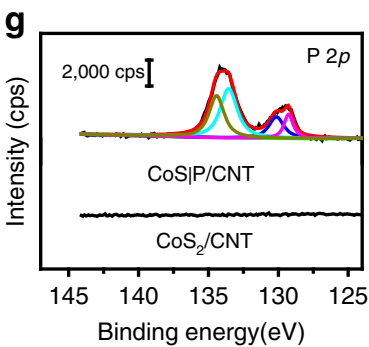

Figure 3 | Composition and chemical analysis for the CoS|P/CNT hybrid material. (a) STEM image recorded by a high-angle annular dark field (HAADF) detector showing CoS|P nanoparticles attached on CNTs. Scale bar, $20 \mathrm{~nm}$. (b) STEM-EDS mapping of CoS|P/CNT catalyst showing the distributions of Co (green), P (yellow) and S (orange) within the nanoparticles closely attached to C (red). Scale bar, $20 \mathrm{~nm}$. (c) EDS spectrum of CoS/P/CNT. (d) Raman spectra of $\mathrm{CoS}_{2} / \mathrm{CNT}$ and CoS|P/CNT. (e-g) Co $2 p, \mathrm{~S} 2 p$ and P $2 p$ core level XPS spectra of CoS|P/CNT and CoS $2 / C N T$.

by EDS. This again corroborates that $\mathrm{P}$ is uniformly distributed within the nanoparticles rather than forming a shell-like structure on the original $\mathrm{CoS}_{2}$ nanoparticles.

Electrocatalytic hydrogen evolution. HER electrocatalytic activity of the CoS $\mid \mathrm{P} / \mathrm{CNT}$ hybrid material was assessed in $0.5 \mathrm{M}$ $\mathrm{H}_{2} \mathrm{SO}_{4}$ aqueous solution. Figure $4 \mathrm{a}$ shows the polarization curve of the $\mathrm{CoS} \mid \mathrm{P} / \mathrm{CNT}$ hybrid material as compared with a benchmark $\mathrm{Pt} / \mathrm{C}$ catalyst at a scan rate of $5 \mathrm{mV} \mathrm{s}^{-1}$. With a mass loading of $1.6 \mathrm{mg} \mathrm{cm}^{-2}$, the $\mathrm{CoS} \mid \mathrm{P} / \mathrm{CNT}$ electrode showed a negligible onset overpotential versus the reversible hydrogen electrode (RHE). The catalytic current density increased rapidly with further cathodic polarization to $10 \mathrm{~mA} \mathrm{~cm}^{-2}, 20 \mathrm{~mA} \mathrm{~cm}^{-2}$ and $100 \mathrm{~mA} \mathrm{~cm}^{-2}$ at overpotentials of $48 \mathrm{mV}, 65 \mathrm{mV}$ and $109 \mathrm{mV}$, respectively (Fig. 4a). An exchange current density of $1.14 \mathrm{~mA} \mathrm{~cm}^{-2}$ and a Tafel slope of $55 \mathrm{mV}$ per decade were derived from the polarization curve (Fig. 4b), suggesting a different HER mechanism from Pt which showed a $30 \mathrm{mV}$ per decade Tafel slope indicative of a Volmer-Tafel mechanism ${ }^{36}$. Such performance represents arguably higher HER catalytic activity than any other Co-based catalyst materials reported in the literature, placing our material at the top of all existing noble-metal-free HER catalyst materials working in acidic media (Supplementary Table 1). Our catalyst also showed high durability for HER catalysis. The initial current density $\left(\sim 45 \mathrm{~mA} \mathrm{~cm}^{-2}\right)$ maintained after $24 \mathrm{~h}$ of continuous hydrogen production (Fig. 4c). Stability for $100 \mathrm{~h}$ of HER catalysis was also confirmed (Supplementary Fig. 5). We also performed 2,000 cycles of cyclic voltammetry between 0.25 and $-0.12 \mathrm{~V}$ versus $\mathrm{RHE}$ on the CoS|P/CNT catalyst. The polarization curves showed negligible shift during the test (Fig. 4d). Chronoamperometry in combination with gas chromatography and mass spectrometry (MS) revealed Faradic efficiency of $\sim 100 \%$ for $\mathrm{H}_{2}$ (Supplementary Fig. 6).

\section{Discussion}

The excellent HER catalytic activity and durability of the CoS $\mid \mathrm{P} /$ CNT hybrid material is a direct outcome of its unique material structure imparted by the three designed chemical reaction steps. The first step of reaction grows $\mathrm{Co}_{3} \mathrm{O}_{4}$ nanoparticles on CNTs, which builds the strong electrical and chemical coupling between the nanoparticles and CNTs ${ }^{37,38}$. Consequently, nanoparticles are anchored on the highly conductive CNT network, which can rapidly transport electrons from external circuit to nanoparticle/ electrolyte interface for hydrogen evolution. This step also brings size control of the nanoparticles, which is essential for increasing the electrochemically active surface area and reducing the electron diffusion length within each nanoparticle.

The second step of reaction converts the $\mathrm{Co}_{3} \mathrm{O}_{4}$ nanoparticles to $\mathrm{CoS}_{2}$ nanoparticles, through which highly active sites for HER catalysis are created. The $\mathrm{CoS}_{2} / \mathrm{CNT}$ material is already as active as the final CoS $\mid \mathrm{P} / \mathrm{CNT}$. At a mass loading of $0.8 \mathrm{mg} \mathrm{cm}^{-2}, \mathrm{HER}$ 

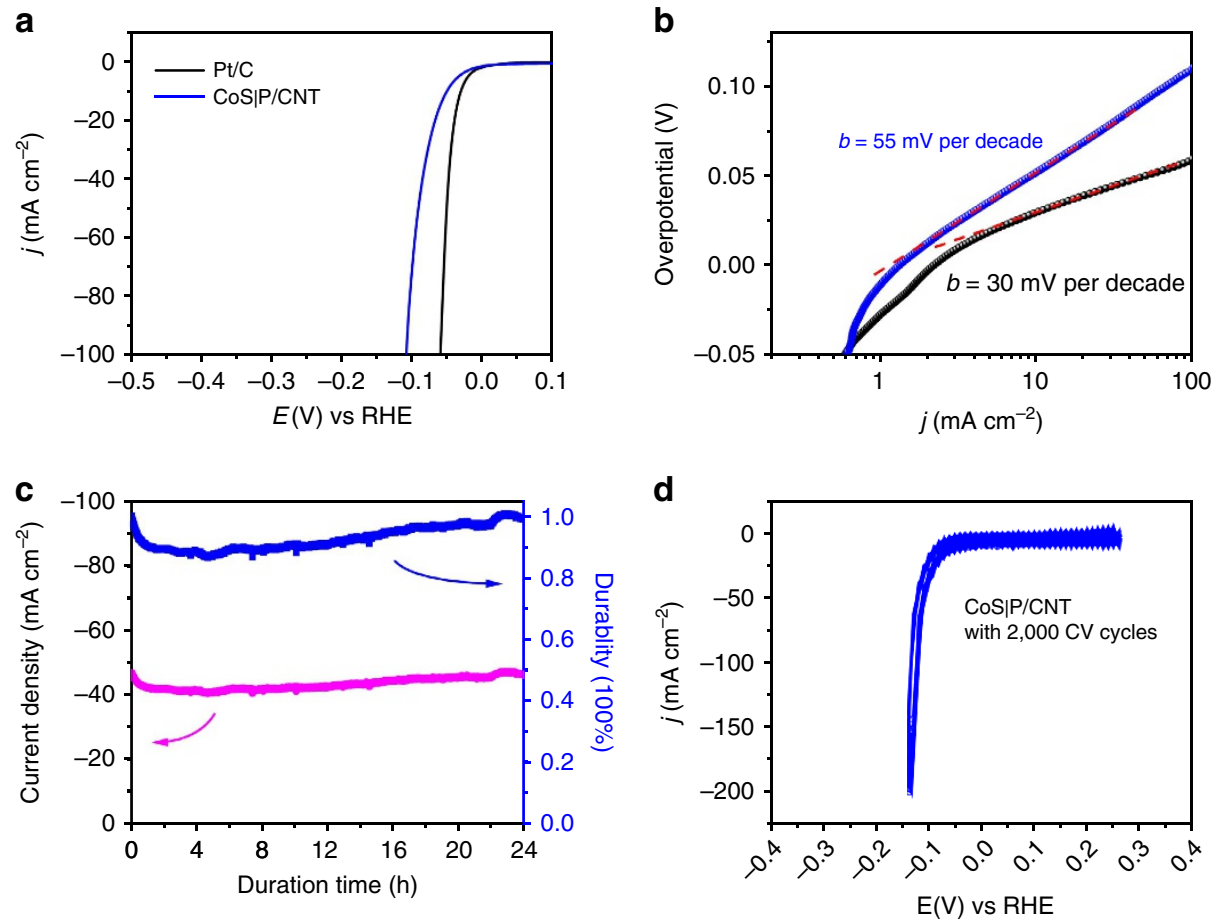

Figure 4 | Electrocatalytic hydrogen evolution over the CoS|P/CNT catalyst. (a) Polarization curves for HER on the CoS|P/CNT hybrid and a commercial $\mathrm{Pt} / \mathrm{C}$ catalyst at $5 \mathrm{mVs}^{-1}$. The catalyst mass loading was $0.4 \mathrm{mg} \mathrm{cm}^{-2}$ for Pt/C catalyst. (b) Tafel plots for the CoS|P/CNT and Pt/C catalysts derived from the polarization curves in a. (c) Chronoamperometric response ( $j \sim t$ curve) recorded on the CoS|P/CNT electrode at a constant overpotential of $95 \mathrm{mV}$ with iR compensation. (d) $\mathrm{CV}$ test between 0.25 and $-0.12 \mathrm{~V}$ versus RHE at a scan rate of $100 \mathrm{mV} \mathrm{s}^{-1}$ for 2,000 cycles. The catalyst mass loading of $\mathrm{CoS} \mid \mathrm{P} / \mathrm{CNT}$ was $1.6 \mathrm{mg} \mathrm{cm}^{-2}$ unless otherwise noted.

current density of $10 \mathrm{mAcm}^{-2}$ was reached at an overpotential of $61 \mathrm{mV}$ for the $\mathrm{CoS}_{2} / \mathrm{CNT}$ as compared with $64 \mathrm{mV}$ for the $\mathrm{CoS} \mid \mathrm{P} / \mathrm{CNT}$ under the same condition (Supplementary Fig. 8a). This already represents one of the most active cobalt chalcogenide HER catalyst materials ${ }^{10,28,39-42}$. The sequential synthetic method, namely oxide growth followed by conversion to sulfide, is responsible for the superior catalytic activity, as corroborated by our control experiment (Supplementary Fig. 7).

In spite of the excellent activity as a result of size control and CNT hybridization, the intrinsic instability of $\mathrm{CoS}_{2}$ in strong acid is exacerbated. The $\mathrm{CoS}_{2} / \mathrm{CNT}$ material is extremely unstable during HER catalysis in $0.5 \mathrm{M} \mathrm{H}_{2} \mathrm{SO}_{4}$. Under constant potential operation, the current density decreased drastically by $70 \%$ in less than $30 \mathrm{~min}$ (Fig. 5a). Such a dramatic deterioration was accompanied by substantial dissolution of the $\mathrm{CoS}_{2}$ active phase into the electrolyte. A Co concentration of $\sim 2.1$ p.p.m. in the electrolyte was measured by inductively coupled plasma MS (ICP-MS) after $30 \mathrm{~min}$ of HER catalysis (Fig. 5b), corresponding to about $17.5 \%$ of the $\mathrm{CoS}_{2}$ having been dissolved. The concentration of dissolved Co gradually increased to $\sim 2.9$ p.p.m. over $20 \mathrm{~h}$ of HER operation. The $\mathrm{CoS}_{2} / \mathrm{CNT}$ material is also sensitive to air and moisture. After being stored in ambient condition for 2 weeks, the $\mathrm{CoS}_{2}$ nanoparticles were completely oxidized to $\mathrm{CoSO}_{4} \cdot 7 \mathrm{H}_{2} \mathrm{O}$ ( $\mathrm{PDF} \# 16-04872$ ), which is soluble in water and HER inactive (Fig. 5c).

The third step of reaction ( $\mathrm{P}$ substitution) is critical to the chemical stability and catalytic durability of the final material (Fig. 5 and Supplementary Fig. 8). No phase change was detected by XRD for the CoS|P/CNTs material after being stored in ambient condition for 2 weeks (Fig. 5c), suggesting significantly improved chemical stability to oxygen and moisture. This is consistent with our XPS results that $\mathrm{P}$ substitution could mitigate sulfate formation on $\mathrm{CoS}_{2}$ surface. The CoS $\mid \mathrm{P} / \mathrm{CNT}$ s catalyst was able to sustain a current density of $\sim 10 \mathrm{~mA} \mathrm{~cm}^{-2}$ during $20 \mathrm{~h}$ of continuous HER operation (Fig. 5a), substantially more stable than the $\mathrm{CoS}_{2} / \mathrm{CNT}$ catalyst under working conditions. In consistency, the amount of Co leaching into the electrolyte was much lower than that for the $\mathrm{CoS}_{2} / \mathrm{CNT}$ catalyst. The Co concentration in electrolyte gradually reached $\sim 0.6$ p.p.m. within $12 \mathrm{~h}$ and did not increase significantly in the following $8 \mathrm{~h}$ (Fig. 5b). To visually demonstrate the stability difference between the $\mathrm{CoS}_{2} / \mathrm{CNT}$ and $\mathrm{CoS} \mid \mathrm{P} / \mathrm{CNT}$ in the electrolyte, we conducted a colorimetric comparison (Supplementary Methods) after the $\mathrm{CoS}_{2} / \mathrm{CNT}$ and $\mathrm{CoS} \mid \mathrm{P} / \mathrm{CNT}$ hybrids were soaked in $0.5 \mathrm{M}$ $\mathrm{H}_{2} \mathrm{SO}_{4}$ for $2 \mathrm{~h}$. The Nitrite $\mathrm{R}$ salt is widely used as a colour indicator to detect cobalt in solution as it can form a red coloured complex with $\mathrm{Co}^{2+}$ ions. The solution in which the $\mathrm{CoS}_{2} / \mathrm{CNT}$ was soaked exhibited an orange colour (Fig. 5d), clearly showing a considerable amount of Co dissolved into the acid. In contrast, the solution in which the $\mathrm{CoS} \mid \mathrm{P} / \mathrm{CNT}$ was soaked remained almost the same colour as the blank control, confirming greatly improved stability of the CoS/P/CNT catalyst against acid corrosion. Our control experiment clearly verified that it is indeed the $\mathrm{P}$ substitution rather than the high-temperature annealing process that renders the excellent catalytic durability (Supplementary Fig. 9).

It is worth emphasizing here that the $\mathrm{P}$ substitution step is only for improving material stability and catalytic durability, but not for enhancing catalytic activity. This is different from the recent study where the CoPS is much more active than the $\mathrm{CoS}_{2}$ with similar morphology ${ }^{26}$. It is also noted that our $\operatorname{CoS} \mid \mathrm{P}$ is likely in a different structure than the recently reported CoPS. The CoPS is a distinct ternary pyrite-type phase featuring $\mathrm{P}-\mathrm{S}$ bonds without any $\mathrm{S}-\mathrm{S}$ bonds as in the $\mathrm{CoS}_{2}$ pyrite structure. The corresponding XRD and Raman peaks significantly shifted as a result of the shrunk lattice parameters. In contrast, our $\operatorname{CoS} \mid \mathrm{P}$ is more likely to 

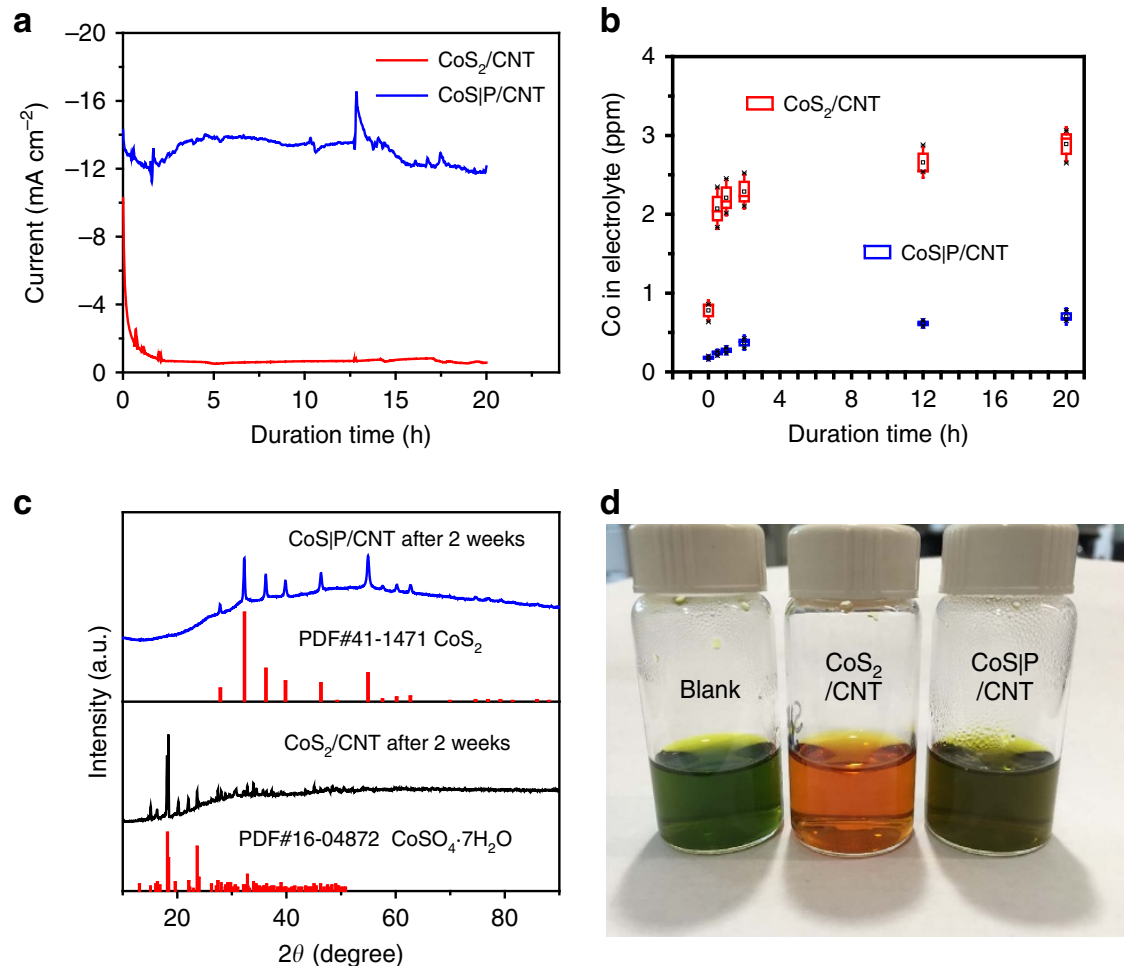

Figure 5 | Comparison of chemical stability and catalytic durability between $\mathbf{C o S}_{\mathbf{2}} / \mathbf{C N T}$ and $\mathbf{C o S} \mid \mathbf{P} / \mathbf{C N T}$. (a) Typical chronoamperometric responses (j t curves) of the $\mathrm{CoS}_{2} / \mathrm{CNT}$ and $\mathrm{CoS} \mid \mathrm{P} / \mathrm{CNT}$ catalysts driving hydrogen evolution at the overpotential of $77 \mathrm{mV}$ without iR compensation for $20 \mathrm{~h}$ in $0.5 \mathrm{M} \mathrm{H}_{2} \mathrm{SO}_{4}$ solution. About $0.4 \mathrm{mg}$ of each catalyst was loaded on a carbon fibre paper with $0.5 \mathrm{~cm}^{2}$ of active area. The sharp current fluctuations were caused by the sampling of electrolyte during the electrolysis process. (b) Box plots (median and quartiles) representing the concentrations of Co dissolved in $20 \mathrm{ml}$ of electrolyte as the HER catalysis proceeds. The vertical whiskers represent the s.d. The statistics are derived from at least three independent measurements. (c) XRD patterns of $\mathrm{CoS}_{2} / \mathrm{CNT}$ and $\mathrm{CoS} / \mathrm{P} / \mathrm{CNT}$ after 2 weeks of storage in ambient conditions. (d) Colorimetric comparison of the $\mathrm{CoS}_{2} /$ CNT and $\mathrm{CoS} / \mathrm{P} / \mathrm{CNT}$ hybrids soaked in $0.5 \mathrm{M} \mathrm{H}_{2} \mathrm{SO}_{4}$ solution for $2 \mathrm{~h}$; Nitrite $\mathrm{R}$ salt was used as the colour indicator.

be a $\mathrm{CoS}_{2}$ pyrite structure (no lattice parameter shrinking) with some of the $\mathrm{S}$ atoms randomly substituted by $\mathrm{P}$ atoms. The structural difference is a direct outcome of the different synthetic methods adopted. The CoPS is prepared by converting $\mathrm{Co}(\mathrm{OH})\left(\mathrm{CO}_{3}\right)_{0.5} \cdot \mathrm{XH}_{2} \mathrm{O}$ with pre-formed $\mathrm{P}_{x} \mathrm{~S}_{y}$, therefore the $\mathrm{P}-\mathrm{S}$ bonding is exclusive and CoPS is the only available composition. In this study, $\mathrm{CoS} \mid \mathrm{P}$ is derived from $\mathrm{CoS}_{2}$ so that the $\mathrm{P} / \mathrm{S}$ ratio in the $\operatorname{CoS}_{2-x} \mathrm{P}_{x} / \mathrm{CNT}$ materials can be readily tuned between 0 and 1 while keeping the mother structure and lattice parameters unchanged (Supplementary Fig. 10). As we gradually increase the substitution level of $\mathrm{P}$, the influence on HER activity is negligible but the catalytic durability improves drastically (Supplementary Fig. 11).

To probe the molecular origins of the structural stability of $\mathrm{CoS} / \mathrm{P} / \mathrm{CNT}$, we performed X-ray absorption near edge structure (XANES) spectroscopy measurements. The absorption edges of the Co K-edge spectra of $\mathrm{CoS}_{2} / \mathrm{CNT}$ and $\mathrm{CoS} / \mathrm{P} / \mathrm{CNT}$ lie close to each other (Fig. 6a), suggesting similar oxidation states of $\mathrm{Co}$ in both materials, which match the XPS results. The relatively small pre-edge peaks of both spectra suggest that the Co ions invariably reside in octahedral coordination environment (Fig. 6c). It is noted that the $\operatorname{CoS} \mid \mathrm{P} / \mathrm{CNT}$ spectrum shows a shoulder peak at $\sim 7,717 \mathrm{eV}$. Similar features have been observed in other systems and are attributed to the covalency effect ${ }^{43}$. This correlates well with the pre-edge absorption intensity difference between the $\mathrm{CoS} \mid \mathrm{P} / \mathrm{CNT}$ and $\mathrm{CoS}_{2} / \mathrm{CNT}$ (inset of Fig. 6a). For both $\mathrm{CoS} \mid \mathrm{P}$ and $\mathrm{CoS}_{2}$, the lowest unoccupied molecular orbitals are the anti-bonding $e_{\mathrm{g}}{ }^{*}$ orbitals $^{4}$. These orbitals are due to hybridization between transition metal $3 d$ and ligand $3 p$ orbitals and are thus very sensitive to covalency. The stronger pre-edge adsorption of $\mathrm{CoS} \mid \mathrm{P} / \mathrm{CNT}$ compared with that of $\mathrm{CoS}_{2} / \mathrm{CNT}$ suggests more $\mathrm{p}$ features in the Co $3 d$ state and therefore stronger covalency for the $\mathrm{CoS} \mid \mathrm{P}$ compound. Stronger covalency between the transition metal and ligands in $\operatorname{CoS} \mid \mathrm{P} /$ CNT is also supported by the S K-edge XANES spectra. As shown in Fig. 6b, the $\mathrm{CoS} / \mathrm{P} / \mathrm{CNT}$ spectrum exhibits a stronger peak at $\sim 2,469 \mathrm{eV}$ than the $\operatorname{CoS}_{2} / \mathrm{CNT}$, corresponding to higher probability of $1 s$ to $e_{\mathrm{g}}{ }^{*}$ transition as a result of stronger covalency of the metal-ligand bonds in $\operatorname{CoS} \mid \mathrm{P}^{45}$.

To further rationalize the experimental findings, we performed first-principles DFT calculations for $\operatorname{CoS}_{2-x} \mathrm{P}_{x}(x=0,0.5,1.0,1.5$ and 2) with both the cubic (pyrite) and monoclinic structures. The results for the relative stability of the two phases are shown in Fig. 6d. We found that $\operatorname{CoS}_{2}$ is more stable in the cubic structure than in the monoclinic structure, but only with a marginal energy difference of about $0.03 \mathrm{eV}$ per formula unit (f.u.). In contrast, $\mathrm{CoP}_{2}$ is substantially more stable in the monoclinic structure by about $0.6 \mathrm{eV}$ per f.u. When $S$ is substituted by $P$, the phosphosulfides would be more stable in the monoclinic structure within a large range of $x$ if we assume a linear dependence of the relative stability of the two phases on the composition. However, first-principles calculations clearly suggest that $\operatorname{CoS}_{2}{ }_{x} \mathrm{P}_{x}$ is more stable in the cubic structure for $x \leq 1.0$, which agrees well with our experimental results. The remarkable stability of the cubic structure can be attributed to the fact that cubic-phase $\mathrm{CoS}_{2}$ and $\mathrm{CoP}_{2}$ have very similar optimized equilibrium volume $\left(V_{0}\right)$ (41.6 versus $40.1 \AA^{3}$ per f.u.). In comparison, the $V_{0}$ difference between monoclinic-phase $\mathrm{CoS}_{2}$ and $\mathrm{CoP}_{2}$ is much larger (42.8 versus $39.2 \AA^{3}$ per f.u.). Substitution of $S$ by $P$ would induce much less strain in the cubic than in the monoclinic structure. 
a

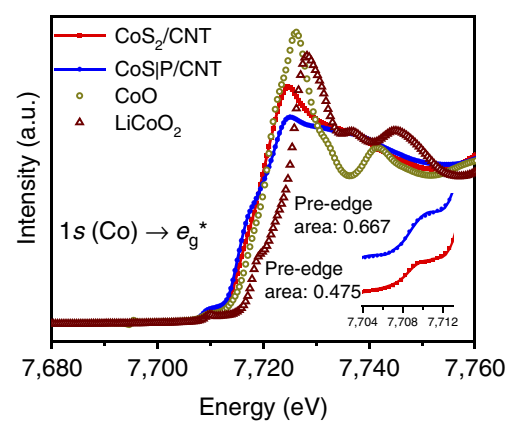

C

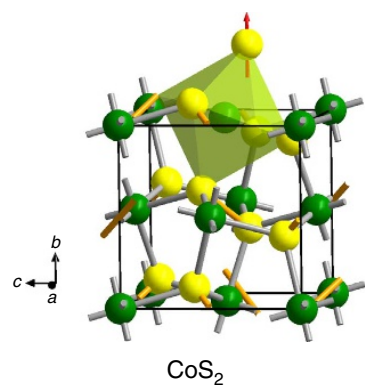

d

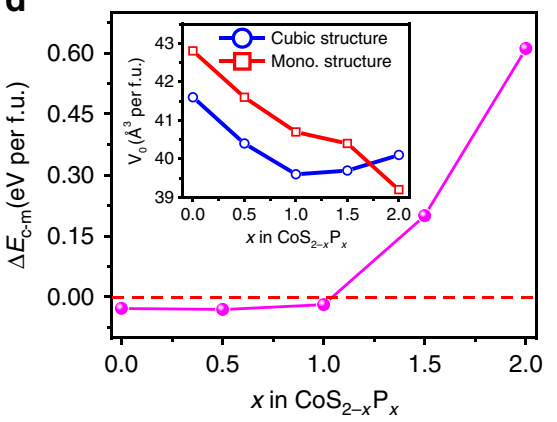

b
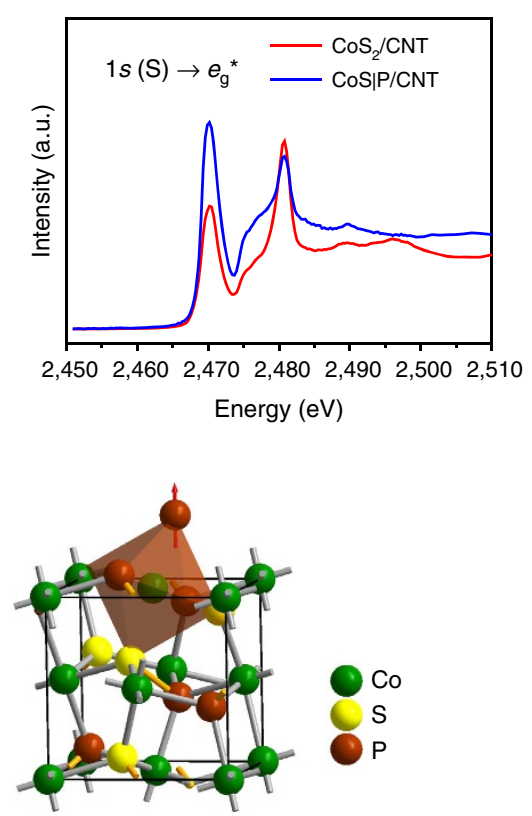

$\operatorname{CoS} \mid \mathrm{P}$

e

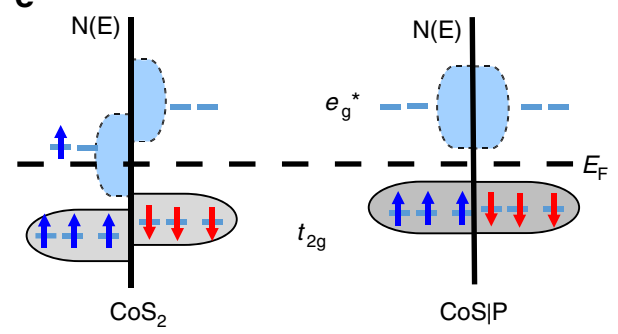

Figure 6 | XANES spectra of CoS|P/CNT and structural stability discussion based on first-principles calculations. (a) Cobalt K-edge XANES spectra of $\mathrm{CoS}_{2} / \mathrm{CNT}$ and $\mathrm{CoS} \mid \mathrm{P} / \mathrm{CNT}$ compared with $\mathrm{Co} \mathrm{ol}^{\mathrm{II}}(\mathrm{CoO})$ and $\mathrm{Co}$ III $\left(\mathrm{LiCoO}_{2}\right)$ standards. Pre-edge features correspond to transition from $1 \mathrm{~s}(\mathrm{Co})$ orbital to $\mathrm{e}_{\mathrm{g}}{ }^{*}$ anti-bonding state, which is metal $3 d$ and ligand $3 p$ hybrid orbitals. The pre-edge features were fitted by two pseudo-Voigt functions with the results shown in the inset graph. (b) Sulfur K-edge XANES spectra of $\operatorname{CoS}_{2} / \mathrm{CNT}$ and $\mathrm{CoS} \mid \mathrm{P} / \mathrm{CNT}$. (c) Structure illustration of pyrite-phase $\operatorname{CoS}_{2}$ and $\operatorname{CoS}_{\mathrm{P}} \mathrm{P}\left(\mathrm{CoS}_{2}-\mathrm{P}_{x_{1}}\right.$ $x=1$ ), each with a representative coordination polyhedron. (d) The energy difference per formula unit (f.u.) between the cubic and monoclinic phases as a function of the P substitution extent obtained from DFT calculations. The inset shows the equilibrium volume per f.u. in the cubic and monoclinic phases as a function of the P substitution extent. (e) Conceptual energy level diagrams of the frontier molecular orbitals for pyrite-phase $\operatorname{CoS}_{2}$ and $\mathrm{CoS}_{\mathrm{P}} \mathrm{P}$ derived from the calculated electronic structures (Supplementary Fig. 12). $\mathrm{CoS}_{2}$ is magnetic at room temperature. As a result spin-up and spin-down electrons have different energy levels. $\operatorname{CoS} \mid \mathrm{P}\left(\mathrm{CoS}_{2-x} \mathrm{P}_{x} x=1\right)$ is non-magnetic.

Therefore, the pyrite-structured $\operatorname{CoS}_{2-x} \mathrm{P}_{x}$ is stable within a quite wide range of $\mathrm{P}$ doping levels $(x \leq 1.0)$.

First-principles calculations can also provide insight into why incorporation of $\mathrm{P}$ improves the chemical stability of pyrite-structured $\mathrm{CoS}_{2}$. From projected density of states analysis (Supplementary Fig. 12), we see that P substitution significantly influences the nature of chemical bonding between Co and S/P. In the pyrite structure, each Co atom is coordinated in an octahedral ligand field (Fig. 6c), and therefore the $3 d$ orbitals are split into $t_{2 \mathrm{~g}}$ and $e_{\mathrm{g}}{ }^{*}$ manifolds that are of non-bonding and anti-bonding characteristics, respectively. As qualitatively demonstrated in Fig. 6e, the highest occupied states in $\mathrm{CoS}_{2}$ are of anti-bonding nature, which is the origin of the instability. When half of the $S$ atoms are replaced by $\mathrm{P}$, which has fewer valence electrons, the anti-bonding $e_{\mathrm{g}}^{*}$ orbitals are depleted, which strengthens the chemical bonding between Co and ligands and thus enhances the chemical stability of the material. This is in good agreement with our XANES results. In conclusion, we demonstrate a novel structure design and synthesis of a highly active and stable HER catalyst material based on pyrite-structured $\operatorname{CoS} \mid \mathrm{P}$. The sequential synthetic strategy we adopt imparts electrical conductivity, catalytic activity and stability to the material. The CoS $\mid \mathrm{P} / \mathrm{CNT}$ catalyst exhibits arguably the highest catalytic activity among all non-noble metal based catalysts. P substitution is critical to chemical stability and catalytic durability of the material. The molecular origins are rationalized by spectroscopy characterization and computational modelling.

\section{Methods}

Material synthesis. The $\mathrm{CoS} \mid \mathrm{P} / \mathrm{CNT}$ hybrid was prepared through a three-step method. In the first step (synthesis of $\mathrm{Co}_{3} \mathrm{O}_{4} / \mathrm{CNT}$ ), $4 \mathrm{mg}$ of mildly oxidized CNTs (the CNTs were oxidized following a modified Hummers method as described in Supplementary Information) were dispersed in $14 \mathrm{ml}$ of ethanol by sonication for $1 \mathrm{~h}$. Then, $0.8 \mathrm{ml}$ of $0.2 \mathrm{M}$ cobalt acetate aqueous solution and $0.6 \mathrm{ml}$ of $\mathrm{NH}_{4} \mathrm{OH}$ 
(30\%) were added to the suspension. The hydrolysis reaction was kept at $80^{\circ} \mathrm{C}$ in oil bath with stirring for $12 \mathrm{~h}$. After that, the product was collected by centrifuge. The precipitate was then washed with ethanol and DI water. The resulting $\mathrm{Co}_{3} \mathrm{O}_{4} / \mathrm{CNT}$ was lyophilized. To prepare $\mathrm{CoS}_{2} / \mathrm{CNT}, 20 \mathrm{mg}$ of $\mathrm{Co}_{3} \mathrm{O}_{4} / \mathrm{CNT}$ was dispersed in $20 \mathrm{ml}$ of DI water by sonication for $40 \mathrm{~min}$, followed by the addition of $0.75 \mathrm{ml}$ of $1 \mathrm{M}$ thioacetamide solution. After that, the reaction mixture was transferred to a $40 \mathrm{ml}$ autoclave for hydrothermal reaction at $200^{\circ} \mathrm{C}$ for $6 \mathrm{~h}$. The resulting product was collected by centrifugation and repeatedly washed with DI water. The $\mathrm{CoS}_{2} / \mathrm{CNT}$ hybrid was then freeze-dried. In the third step, $5 \mathrm{mg}$ of $\mathrm{CoS}_{2} / \mathrm{CNT}$ and $100 \mathrm{mg}$ of $\mathrm{NaH}_{2} \mathrm{PO}_{2} \cdot \mathrm{H}_{2} \mathrm{O}$ were placed at two separate positions in a ceramic crucible with the $\mathrm{NaH}_{2} \mathrm{PO}_{2} \cdot \mathrm{H}_{2} \mathrm{O}$ at the upstream side. The samples were heated at $400^{\circ} \mathrm{C}$ for $1 \mathrm{~h}$ with $\mathrm{Ar}$ gas flowing at 200 s.c.c.m. The final product contains about $60 \mathrm{wt} \%$ of $\mathrm{CoS} \mid \mathrm{P}$ and $40 \mathrm{wt} \%$ of CNTs.

Electrochemical measurements. To prepare catalyst ink, $1 \mathrm{mg}$ of $\operatorname{CoS} \mid \mathrm{P} / \mathrm{CNT}$ was mixed with $190 \mu \mathrm{l}$ of water, $50 \mu \mathrm{l}$ of ethanol and $10 \mu \mathrm{l}$ of $5 \mathrm{wt} \%$ Nafion solution by sonication for $1 \mathrm{~h}$. Subsequently, 50-200 $\mu \mathrm{l}$ of the catalyst ink was drop-dried onto a carbon fibre paper (Spectracarb 2050 A from Fuel Cell Store) to cover an area of $0.5 \mathrm{~cm}^{2}\left(0.4-1.6 \mathrm{mg} \mathrm{cm}^{-2}\right)$. The electrode was further heated at $90^{\circ} \mathrm{C}$ in vacuum for $2 \mathrm{~h}$. HER catalytic measurements were performed with a CHI 760D electrochemistry workstation (CH instruments, USA). A conventional three electrode cell configuration was employed. A saturated calomel electrode (SCE) was used as the reference electrode, and a graphite rod was used as the counter electrode. $0.5 \mathrm{M} \mathrm{H}_{2} \mathrm{SO}_{4}$ solution was used as electrolyte. Linear sweep voltammetry was recorded at a scan rate of $5 \mathrm{mV} \mathrm{s}^{-1}$. All the polarization curves were iR-corrected. The reference electrode was calibrated against the RHE as shown in Supplementary Fig. 13. All the potentials reported in our work were converted according to $\mathrm{E}$ (versus RHE) $=\mathrm{E}$ (versus SCE) $+0.278 \mathrm{~V}$.

First-principles calculations. All DFT calculations were conducted by using the Vienna $a b$ initio simulation package (VASP) suite that is based on the projectoraugmented wave (PAW) approach and the plane wave basis set ${ }^{46}$. The structures of $\operatorname{CoS}_{2-x} \mathrm{P}_{x}(x=0,0.5,1.0,1.5$ and 2$)$, including lattice constants and internal coordinates, in both the cubic (pyrite) and monoclinic phases, were optimized using the Perdew-Burke-Ernzerhof ${ }^{47}$ approximation for the exchange-correlation functional with a plane wave energy cutoff of $400 \mathrm{eV}$, which is about 1.5 times of the default cutoff value such that the Pulay stress problem can be avoided. To account for the possible strong correlation effects in the Co $3 d$ electrons, the energy difference between the cubic and monoclinic phases was calculated using the PBE plus the Hubbard $U$ correction $(\mathrm{PBE}+U$ ) approach in the rotationally invariant scheme (LDAUTYPE $=1$ ) ${ }^{48}$ with $U=4.5 \mathrm{eV}$ and $J=0.5 \mathrm{eV}$. We used the special quasi-random structure approach ${ }^{49}$ implemented in the ATAT code ${ }^{50}$ to model the random substitution of $\mathrm{S}$ by $\mathrm{P}$ in $\operatorname{CoS}_{2-x} \mathrm{P}_{x}$ using a supercell of 24 atoms.

\section{References}

1. Turner, J. A. Sustainable hydrogen production. Science 305, 972-974 (2004).

2. Mazloomi, K. \& Gomes, C. Hydrogen as an energy carrier: Prospects and challenges. Renew. Sust. Energ. Rev. 16, 3024-3033 (2012).

3. Yang, J. \& Shin, H. S. Recent advances in layered transition metal dichalcogenides for hydrogen evolution reaction. J. Mater. Chem. A 2, 5979-5985 (2014).

4. Faber, M. S. \& Jin, S. Earth-abundant inorganic electrocatalysts and their nanostructures for energy conversion applications. Energy Environ. Sci. 7, 3519-3542 (2014).

5. Zou, X. \& Zhang, Y. Noble metal-free hydrogen evolution catalysts for water splitting. Chem. Soc. Rev. 44, 5148-5180 (2015).

6. Jaramillo, T. F. et al. Identification of active edge sites for electrochemical $\mathrm{H}_{2}$ evolution from $\mathrm{MoS}_{2}$ nanocatalysts. Science 317, 100-102 (2007).

7. Laursen, A. B., Kegnaes, S., Dahl, S. \& Chorkendorff, I. Molybdenum sulfidesefficient and viable materials for electro - and photoelectrocatalytic hydrogen evolution. Energy Environ. Sci. 5, 5577-5591 (2012).

8. Voiry, D. et al. Enhanced catalytic activity in strained chemically exfoliated $\mathrm{WS}_{2}$ nanosheets for hydrogen evolution. Nat. Mater. 12, 850-855 (2013).

9. Wang, H. et al. $\mathrm{MoSe}_{2}$ and $\mathrm{WSe}_{2}$ nanofilms with vertically aligned molecular layers on curved and rough surfaces. Nano Lett. 13, 3426-3433 (2013).

10. Kong, D., Wang, H., Lu, Z. \& Cui, Y. CoSe $e_{2}$ nanoparticles grown on carbon fibre paper: an efficient and stable electrocatalyst for hydrogen evolution reaction. J. Am. Chem. Soc. 136, 4897-4900 (2014).

11. McEnaney, J. M. et al. Amorphous molybdenum phosphide nanoparticles for electrocatalytic hydrogen evolution. Chem. Mater. 26, 4826-4831 (2014).

12. Jiang, P. et al. A cost-effective 3D hydrogen evolution cathode with high catalytic activity: $\mathrm{FeP}$ nanowire array as the active phase. Angew. Chem. Int. Ed. 53, 12855-12859 (2014).

13. Liu, Q. et al. Carbon nanotubes decorated with CoP nanocrystals: a highly active non-noble-metal nanohybrid electrocatalyst for hydrogen evolution. Angew. Chem. Int. Ed. 53, 6710-6714 (2014).
14. Li, Y. H. et al. Local atomic structure modulations activate metal oxide as electrocatalyst for hydrogen evolution in acidic water. Nat. Commun. 6, 8064 (2015).

15. Yan, H. J. et al. Phosphorus-modified tungsten nitride/reduced graphene oxide as a high-performance, non-noble-metal electrocatalyst for the hydrogen evolution reaction. Angew. Chem. Int. Ed. 54, 6325-6329 (2015).

16. Kong, D. et al. Synthesis of $\mathrm{MoS}_{2}$ and $\mathrm{MoSe}_{2}$ films with vertically aligned layers. Nano Lett. 13, 1341-1347 (2013).

17. Li, Y. G. et al. $\mathrm{MoS}_{2}$ nanoparticles grown on graphene: an advanced catalyst for the hydrogen evolution reaction. J. Am. Chem. Soc. 133, 7296-7299 (2011).

18. Yang, Y., Fei, H., Ruan, G., Xiang, C. \& Tour, J. M. Edge-oriented $\mathrm{MoS}_{2}$ nanoporous films as flexible electrodes for hydrogen evolution reactions and supercapacitor devices. Adv. Mater. 26, 8163-8168 (2014).

19. Voiry, D. et al. Conducting $\mathrm{MoS}_{2}$ nanosheets as catalysts for hydrogen evolution reaction. Nano Lett. 13, 6222-6227 (2013).

20. Morales-Guio, C. G. \& Hu, X. Amorphous molybdenum sulfides as hydrogen evolution catalysts. Acc. Chem. Res. 47, 2671-2681 (2014).

21. Kibsgaard, J. \& Jaramillo, T. F. Molybdenum phosphosulfide: an active, acid-stable, earth-abundant catalyst for the hydrogen evolution reaction. Angew. Chem. Int. Ed. 53, 14433-14437 (2014).

22. Deng, J. et al. Triggering the electrocatalytic hydrogen evolution activity of the inert two-dimensional $\mathrm{MoS}_{2}$ surface via single-atom metal doping. Energy Environ. Sci. 8, 1594-1601 (2015).

23. Wang, H. et al. Electrochemical tuning of vertically aligned $\mathrm{MoS}_{2}$ nanofilms and its application in improving hydrogen evolution reaction. Proc. Natl Acad. Sci. USA 110, 19701-19706 (2013).

24. Wang, T. et al. Electrochemically fabricated polypyrrole and $\mathrm{MoS}_{\mathrm{x}}$ copolymer films as a highly active hydrogen evolution electrocatalyst. Adv. Mater. 26, 3761-3766 (2014).

25. Gao, M. R. et al. An efficient molybdenum disulfide/cobalt diselenide hybrid catalyst for electrochemical hydrogen generation. Nat. Commun. 6, 5982 (2015).

26. Caban-Acevedo, M. et al. Efficient hydrogen evolution catalysis using ternary pyrite-type cobalt phosphosulphide. Nat. Mater. 14, 1245-1251 (2015).

27. Zhang, H. et al. Highly crystallized cubic cattierite $\mathrm{CoS}_{2}$ for electrochemically hydrogen evolution over wide $\mathrm{pH}$ range from 0 to 14 . Electrochim. Acta 148, 170-174 (2014).

28. Faber, M. S. et al. High-performance electrocatalysis using metallic cobalt pyrite $\mathrm{CoS}_{2}$ micro- and nanostructures. J. Am. Chem. Soc. 136, 10053-10061 (2014).

29. Anastassakis, E. Light scattering and ir measurements in $\mathrm{XS}_{2}$ pryite-type compounds. J. Chem. Phys. 64, 3604 (1976).

30. Lyapin, S. et al. Raman studies of nearly half-metallic ferromagnetic $\mathrm{CoS}_{2}$. J. Phys. Condens. Matter 26, 396001 (2014).

31. Shadike, Z., Cao, M. H., Ding, F., Sang, L. \& Fu, Z. W. Improved electrochemical performance of $\mathrm{CoS}_{2}$-MWCNT nanocomposites for sodium-ion batteries. Chem. Commun. 51, 10486-10489 (2015).

32. Ma, L. B. et al. CoP nanoparticles deposited on reduced graphene oxide sheets as an active electrocatalyst for the hydrogen evolution reaction. J. Mater. Chem. A 3, 5337-5343 (2015).

33. Jiang, J. et al. Synthesis of $\mathrm{FeP}_{2} / \mathrm{C}$ nanohybrids and their performance for hydrogen evolution reaction. J. Mater. Chem. A 3, 499-503 (2015).

34. $\mathrm{Pu}, \mathrm{Z}$., Liu, Q., Asiri, A. M. \& Sun, X. Tungsten phosphide nanorod arrays directly grown on carbon cloth: a highly efficient and stable hydrogen evolution cathode at all pH values. ACS Appl. Mater. Interfaces 6, 21874-21879 (2014).

35. Xiao, M., Miao, Y., Tian, Y. \& Yan, Y. Synthesizing Nanoparticles of Co-P-Se compounds as electrocatalysts for the hydrogen evolution reaction. Electrochim. Acta 165, 206-210 (2015).

36. Saraby-Reintjes, A. Kinetic criteria for the mechanism of the hydrogen evolution reaction. Electrochim. Acta. 31, 251-254 (1986).

37. Fan, Q., Liu, W., Weng, Z., Sun, Y. \& Wang, H. Ternary hybrid material for high-performance lithium-sulfur battery. J. Am. Chem. Soc. 137, 12946-12953 (2015).

38. Weng, Z. et al. Metal/oxide interface nanostructures generated by surface segregation for electrocatalysis. Nano Lett. 15, 7704-7710 (2015).

39. Kong, D., Cha, J. J., Wang, H., Lee, H. R. \& Cui, Y. First-row transition metal dichalcogenide catalysts for hydrogen evolution reaction. Energy Environ. Sci. 6 , 3553-3558 (2013).

40. Peng, S. et al. Cobalt sulfide nanosheet/graphene/carbon nanotube nanocomposites as flexible electrodes for hydrogen evolution. Angew. Chem. Int. Ed. 53, 12594-12599 (2014).

41. Zhang, H. et al. A metallic $\operatorname{CoS}_{2}$ nanopyramid array grown on 3D carbon fibre paper as an excellent electrocatalyst for hydrogen evolution. J. Mater. Chem. A 3, 6306-6310 (2015). 
42. Liu, Q. et al. $\mathrm{CoSe}_{2}$ nanowires array as a $3 \mathrm{D}$ electrode for highly efficient electrochemical hydrogen evolution. ACS Appl. Mater. Interfaces 7, 3877-3881 (2015).

43. Gyu Kim, M., Sang Cho, H. \& Hyun Yo, C. Fe K-edge X-Ray absorption (XANES/EXAFS) spectroscopic study of the nonstoichiometric $\mathrm{SrFe}_{1-\mathrm{x}} \mathrm{Sn}_{\mathrm{x}} \mathrm{O}_{3-\mathrm{y}}$ system. J. Phys. Chem. Solids 59, 1369-1381 (1998).

44. Bither, T. A., Bouchard, R. J., Cloud, W. H., Donohue, P. C. \& Siemons, W. J. Transition metal pyrite dichalcogenides high-pressure synthesis and correlation of properties. Inorg. Chem. 7, 2208-2220 (1968).

45. Sugiura, C. Sulfur-K X-ray absorption-spectra of FeS, $\mathrm{FeS}_{2}$, and $\mathrm{Fe}_{2} \mathrm{~S}_{3}$. J. Chem. Phys. 74, 215-217 (1981).

46. Kresse, G. \& Furthmuller, J. Efficiency of $a b$ initio total energy calculations for metals and semiconductors using a plane-wave basis set. Comput. Mater. Sci. 6, 15-50 (1996).

47. Perdew, J. P., Burke, K. \& Ernzerhof, M. Generalized gradient approximation made simple. Phys. Rev. Lett. 77, 3865-3868 (1996).

48. Liechtenstein, A. I., Anisimov, A. I. \& Zaanen, J. Density-functional theory and strong interactions: orbital ordering in mott-hubbard insulators. Phys. Rev. B Condens. Matter 52, R5467-R5470 (1995).

49. Wei, S., Ferreira, L. G., Bernard, J. E. \& Zunger, A. Electronic properties of random alloys: special quasirandom structures. Phys. Rev. B Condens Matter 42, 9622-9649 (1990).

50. van de Walle, A. Multicomponent multisublattice alloys, nonconfigurational entropy and other additions to the Alloy Theoretic Automated Toolkit. Calphad J. 33, 266-278 (2009).

\section{Acknowledgements}

The work is partially supported by the Yale University and the Global Innovation Initiative from Institute of International Education. The work at BNL was supported by the US Department of Energy, the Assistant Secretary for Energy Efficiency and Renewable Energy, Office of Vehicle Technologies under Contract Number DESC0012704. We acknowledge technical support from the scientists at beamlines 9-BM-B and 12-BM-B of APS (ANL), supported by the U.S. DOE under Contract No. DE-AC02-06CH11357. M.L. and E.I.A. acknowledge the support of the US Department of Energy through Basic Energy Sciences grant DE-FG02-98ER14882 and the use of facilities supported by the National Science Foundation through the Yale Materials Research Science and Engineering Center (Grant No. MRSEC DMR-1119826). H. J. acknowledges the financial support of National Natural Science Foundation of China (Projects No. 1373017 and 21321001). We thank Prof. Fei Wei (Tsinghua University) for providing the CNTs. We appreciate acquisition of XPS spectra by Baowen Li (CMCM IBS Center, the Ulsan National University of Science and Technology).

\section{Author contributions}

W.L. and H.W. conceived the research. W.L. and Y.X. synthesized the materials and performed the electrochemical measurements. E.H. performed the XANES measurements. H.J. performed the DFT calculations. W.L., Z.W., M.L. and Q.F. performed the material characterizations. All authors discussed and analysed the data. W.L., E.H., H.J. and H.W. wrote the paper. All authors discussed and commented on the manuscript.

\section{Additional information}

Supplementary Information accompanies this paper at http://www.nature.com/ naturecommunications

Competing financial interests: The authors declare no competing financial interests.

Reprints and permission information is available online at http://npg.nature.com/ reprintsandpermissions/

How to cite this article: Liu, W. et al. A highly active and stable hydrogen evolution catalyst based on pyrite-structured cobalt phosphosulfide. Nat. Commun. 7:10771 doi: $10.1038 /$ ncomms10771 (2016).

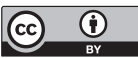

This work is licensed under a Creative Commons Attribution 4.0 International License. The images or other third party material in this article are included in the article's Creative Commons license, unless indicated otherwise in the credit line; if the material is not included under the Creative Commons license, users will need to obtain permission from the license holder to reproduce the material. To view a copy of this license, visit http://creativecommons.org/licenses/by/4.0/ 